\title{
An XPS and ToF-SIMS Investigation of the Outermost Nanometres of a Poly(vinylidene difluoride) Coating.
}

Steven J. Hinder ${ }^{1 *}$, Chris Lowe ${ }^{2}$, and John F. Watts ${ }^{1}$

1 The Surface Analysis Laboratory, School of Engineering, University of Surrey, Guildford, Surrey GU2 7XH, UK.

2 Becker Industrial Coatings Ltd, Goodlass Road, Speke, Liverpool L24 9HJ, UK.

*Correspondence to: Dr Steven J. Hinder, The Surface Analysis Laboratory, School of Engineering (H6), University of Surrey, Guildford, Surrey GU2 7XH, UK.

Email: s.hinder@ surrey.ac.uk Tel: +44 (0) 1483 689624, Fax: +44 (0) 1483686291. 


\begin{abstract}
:
The outermost nanometres of a poly(vinylidene difluoride) (PVdF) based coil coating have been investigated using X-ray photoelectron spectroscopy (XPS) and time-offlight secondary ion mass spectrometry (ToF-SIMS). A reference PVdF based coating formulation and three variations of this formulation were characterised by XPS. The addition of flow agent and/or acrylic co-polymers induced significant changes in the elemental and chemical composition of the coating's air/coating surface. The XPS results indicate that both the flow agent and acrylic co-polymers segregate towards the coating's air/coating surface. The XPS results also suggest that in the fully formulated coating, segregation of the flow agent and co-polymers results in the formation of a surface/sub-surface acrylic layer of sufficient thickness to mask the fluorine signal originating from $\mathrm{PVdF}$.
\end{abstract}

Using a Buckminsterfullerene $\left(\mathrm{C}_{60}\right)$ ion source as an etch source for polymers, depth profiles were obtained of the outermost nanometres of the fully formulated PVdF coating. Fluorine and oxygen depth profiles revealed compositional changes in the coating with respect to depth. The oxygen depth profile revealed the presence of an oxygen rich sub-surface layer within the coating. Molecular depth profiles, acquired for fragment ions diagnostic of the PVdF and acrylic co-polymer components of the coating formulation, revealed the presence of an acrylic co-polymers rich layer in the coating's sub-surface region. All depth profiles suggest that the PVdF coating bulk possesses a homogeneous material composition. The XPS and SIMS results suggest the fully formulated coating is composed of three distinct layers; a thin flow agent 
layer at the air/coating surface, an acrylic co-polymer rich sub-surface layer and the coating bulk.

\section{Keywords :}

Time-of-Flight Secondary Ion Mass Spectrometry; X-ray Photoelectron

Spectroscopy; PVdF coating; Depth Profiling; $\mathrm{C}_{60}$; Polymer. 


\section{Introduction :}

Coil coatings are considered to be one of the most technologically advanced of all coatings/paint systems. As such they must possess a wide range of diverse properties. In the sheet forming processes that the coated stock undergoes, coil coatings are able to withstand, without cracking or loss of coating-metal adhesion, the severe mechanical deformations applied. Coil coatings are also able to resist corrosion and photodegradation for 20 years or more. Steel and aluminium substrates protected by coil coatings have a wide range of commercial and industrial uses. Applications include architectural cladding, caravans, household/domestic appliances and agricultural machinery.

PVdF based coil coatings have seen increasing use in the protection of structures exposed to the environment. The strength of the $\mathrm{C}-\mathrm{F}$ bond ensures that $\mathrm{PVdF}$ molecules are unreactive towards many aggressive environments. The bulk polymer is capable of yielding under applied stress rather than breaking. Finally the electrons in the C-F bond can only be excited by the very short wavelength UV light not found terrestrially as a result of the ozone layer. PVdF based coil coatings follow the contours of formed metal during the forming process without cracking except on the most severe bends. They are resistant to chemical attack and have good gloss retention when they are exposed to UV light. However, PVdF coil coatings typically require that the PVdF component be blended with a secondary polymer, often poly(methyl methacrylate) (PMMA), so as to optimise the performance of the coating [1]. Experimental research has established that the surface composition and structure 
of a polymer blend is generally different to that of the bulk $[2,3]$, presumably as a result of Gibbsian segregation because of differences in the free energy of the different polymeric components within a formulation. Thus a greater understanding of the nature of the surface and sub-surface regions of coatings based on mixed polymer systems, such as those found in PVdF/acrylic co-polymer coatings, will greatly assist the coatings formulator in areas such as intercoat adhesion and weathering.

The use of XPS to investigate PVdF based materials is well documented. Areas of particularly strong research interest in PVdF materials includes PVdF based membranes and PVdF as a biocompatible material. The XPS analyses of PVdF based membranes has generated much research interest in areas such as drug permeation [4], modifying membrane chemical reactivity [5] and microfiltration [6]. PVdF has also received increasing attention as a biocompatible polymer due to its durability, chemical inertness and lack of toxicity. However, due to the lack of functional groups on a PVdF surface, routes to functionalise PVdF and immobilise molecules on its surface have had to be developed. Biomolecules have been immobilised on PVdF surfaces (and characterised by XPS) to improve cell adhesion [7] and to improve biocompatibility [8]. Conversely, procedures to minimise protein interactions with PVdF surfaces have also been developed [9]. The use of XPS to investigate phenomena associated with PVdF based coating formulations has also been reported. Hinder et al have investigated a variety of topics including intercoat adhesion between a PVdF topcoat and a poly(urethane) primer [10], the migration and 
segregation of a silicone additive through a PVdF topcoat [11] and the investigation of a PVdF topcoat/poly(urethane) primer interface buried $20 \mu \mathrm{m}$ beneath a PVdF topcoats air/coating surface [12].

It has long been a desire of polymer and organic coatings scientists to perform depth profiling experiments on polymeric/organic materials using analytical techniques such as ToF-SIMS. However, ion induced damage to polymeric (and other organic) surfaces, typically leads to a loss of chemical and molecular specificity with respect to depth within depth profile layers. Metallic and inorganic materials however are routinely investigated using ToF-SIMS depth profiling techniques. Recently, with the availability of cluster ion based etch sources, the capability to perform ToF-SIMS depth profiling of a number of polymeric (or organic) materials has been possible. The use of a $\mathrm{SF}_{5}{ }^{+}$cluster ion as a depth profiling etch tool for use on polymeric samples has been demonstrated by Fuoco et al employing PMMA thin film on silicon [13], Norrman et al also used PMMA and poly(vinyl chloride) films spin coated onto Si wafer [14] and by Mahoney et al who investigated poly( $L$-lactic acid) (PLLA) doped with 4-acetaminodophenol [15] and PLLA blended with a triblock co-polymer [16]. Most recently, with the introduction of the Buckminsterfullerene $\left(\mathrm{C}_{60}\right)$ cluster ion source as an etch tool, the depth profiling of polymeric (or organic) materials has taken great strides forward. Much of the development work of the $\mathrm{C}_{60}$ ion source as a depth profiling etch tool has been undertaken by Winograd and his colleagues.

Winograd has demonstrated $\mathrm{C}_{60}$ depth profiling of peptides in a trehalose matrix [17], 
Langmuir-Blodgett films [18,19], histamine in an ice matrix [20,21] and PMMA on silicon wafer as a model polymeric system [22].

In this paper, changes in the material composition and chemistry of the surface and sub-surface regions of a PVdF based coating formulation have been investigated. Four variations of a PVdF based coating formulations air/coating surfaces have been analysed using XPS. ToF-SIMS, using a $\mathrm{C}_{60}$ etch source, has been employed to obtain elemental and molecular depth profiles through the outermost nanometres of the coating enabling compositional changes from the air/coating surface, through the subsurface region and down into the bulk of the coating have been investigated.

\section{Experimental :}

\section{Materials and Methods}

The PVdF coating formulations used in this work were applied to aluminium substrate. The PVdF coatings employed were built up stepwise from one containing mainly PVdF to one containing many of the components used in a real world formulation. The 'real world' coating formulation is principally composed of a PVdF resin blended with acrylic co-polymers. The fluoropolymer provides high durability performance and chemical resistance, whilst the acrylics enhance the film forming properties and hardness. The PVdF coating formulations employed in these studies were clear coat formulations containing no pigmentation. The formulation variations employed are described in Table 1. The PVdF coating was applied so as to obtain a 
dry film thickness of $20-22 \mu \mathrm{m}$ and cured by stoving with an oven dwell time of $\sim 30 \mathrm{~s}$ at a peak metal temperature of $\sim 249^{\circ} \mathrm{C}$.

The samples analysed were cured PVdF based coatings applied to aluminium panels $(\sim 16 \mathrm{~cm} \times 10 \mathrm{~cm} \times 0.5 \mathrm{~cm})$. For XPS characterisation of the coating surfaces a disc $\sim 1 \mathrm{~cm}$ in diameter was punched from the sample panel and analysed immediately. To prepare specimens for ToF-SIMS analysis samples $\sim 1 \mathrm{~cm}^{2}$ were cut from the panel using an industrial guillotine.

\section{Surface Analysis by XPS.}

XPS analyses were performed on a Thermo VG Scientific (East Grinstead, UK)

Sigma Probe spectrometer. The instrument employs a monochromated AlK $\alpha$ X-ray source $(\mathrm{hv}=1486.6 \mathrm{eV})$ which was used at a power of $140 \mathrm{~W}$. The area of analysis was approximately $500 \mu \mathrm{m}$ diameter for the coating surfaces analysed. The pass energy was set at $20 \mathrm{eV}$ for core level high-resolution spectra of all elements of interest and at $100 \mathrm{eV}$ for all survey spectra. Charge compensation was achieved using an electron flood gun. The coating samples were held in place on the instruments sample stage by a sprung $\mathrm{Cu} / \mathrm{Be}$ clip. Quantitative surface chemical analyses were calculated from the high resolution core level spectra, following the removal of a non-linear Shirley background. The manufacturer's Avantage software was used which incorporates the appropriate sensitivity factors and corrects for the electron energy analyser transmission function. 


\section{Surface Analysis by ToF-SIMS.}

ToF-SIMS analyses were carried out on an ION-TOF GmbH (Münster, Germany)

TOF.SIMS 5 system. The instrument is equipped with a reflectron type analyser and microchannel plate detector with $20 \mathrm{kV}$ post-acceleration capability. A Bi liquid metal ion source (LMIS) was employed for mass data acquisition. Mass data was acquired using the $\mathrm{Bi}_{3}{ }^{+}$cluster ion. Such clusters ions provide superior secondary ion yields to those available from the more conventional $\mathrm{Ga}^{+}$source. Mass data acquisition was performed by raster scanning over a $75 \times 75 \mu \mathrm{m}^{2}$ area. The area analysed was at the centre of the etch crater formed using the rastered $\mathrm{C}_{60}$ beam. A $25 \mathrm{keV} \mathrm{Bi}_{3}{ }^{+}$primary ion beam delivering $0.8 \mathrm{pA}$ of current was used. A cycle time of $100 \mu \mathrm{s}$ was employed for mass data acquisition. A Buckminsterfullerene ion source was employed to etch the polymeric coating. The $\mathrm{C}_{60}{ }^{+}$ion was used for etching in the depth profiling experiments described here. The $\mathrm{C}_{60}{ }^{+}$etch area was $300 \times 300 \mu \mathrm{m}^{2}$. A $10 \mathrm{kV} \mathrm{C}_{60}{ }^{+}$ primary ion beam with a fluence of $4 \times 10^{14}$ ions $\mathrm{cm}^{-2}$ was employed. An etch interval of $10 \mathrm{~ms}$ was used for all of the depth profile studies described here. Charge compensation was achieved using a low energy electron flood gun for a duration of $20 \mathrm{~ms}$. The depth profiling analyses were performed in the IONTOF 'non-interlaced' mode of operation. That is, each depth profiling cycle starts with mass data acquisition by the $\mathrm{Bi}_{3}{ }^{+}$LMIS; this is then is followed by etching of the coating sample using the $\mathrm{C}_{60}$ source, finally charge compensation using low energy electron flooding completes the depth profiling cycle. 


\section{Results and Discussion :}

Today's coil coating formulation is a complex mix, typically comprising some 15-20 different components. The majority of the formulation comprises resins, co-polymers and solvents. However, many additives including pigments, dispersants, flow-aids and matting agents will be added to the formulation to provide unique properties. In Figure 1 the $250-700 \mathrm{eV}$ binding energy region taken from XPS survey spectra of PVdF based sample coatings, S1-S4 (see Table 1) respectively, are presented. This region of the survey spectrum contains the $\mathrm{C} 1 \mathrm{~s}, \mathrm{O} 1 \mathrm{~s}$ and F1s XPS peaks, these being the only elements present in the S1-S4 coating formulations investigated. Figure 1a contains the C1s (51.5 atomic \%), O1s (2.6 atomic \%) and F1s (45.9 atomic \%) XPS peaks for the S1 coating (PVdF and plasticiser). It is observed in Figure 1a that the F1s peak $(\sim 686 \mathrm{eV})$ is the most intense peak in the spectrum and that the O1s peak $(\sim 531 \mathrm{eV})$ is the least intense. The $\mathrm{C} 1 \mathrm{~s}$ peak in Figure 1a is observed to possess two distinct component peaks. The $\mathrm{C} 1 \mathrm{~s}$ component peak at lower binding energy ( 285 $\mathrm{eV}$ ) is attributed to the hydrocarbon components within the coating materials. The C1s component peak at higher binding energy $(\sim 291 \mathrm{eV})$ in Figure 1a is attributed to the $\mathrm{CF}_{2}$ function of the PVdF [23]. As indicated above there were only two nonsolvent components added to the S1 coating; PVdF and plasticiser. By comparing the actual measured atomic concentrations to theoretical values calculated from a knowledge of the components (Table 2), and further comparing to the calculated values of the "pure' components in Table 3, it is clear that the surface is not composed of a stoichiometric mixture of plasticiser and PVdF. However the fact that the oxygen 
concentration is almost 'correct' indicates that either the PVdF or the plasticiser or both are made up of components with more carbon in them.

In Figure $1 \mathrm{~b}$ the $\mathrm{C} 1 \mathrm{~s}, \mathrm{O} 1 \mathrm{~s}$ and $\mathrm{F} 1 \mathrm{~s}$ peaks for the $\mathrm{S} 2$ coating formulation ( $\mathrm{S} 1$ base formulation plus acrylic flow agent) are presented in the same way they were for the S1 coating sample in Figure 1a. However, for the S2 coating the intensity of the F1s peak (36.3 atomic \%) is much diminished while the intensity of the O1s peak (5.1 atomic \%) has increased, when compared to the S1 reference formulation (see Table 2). There are also significant differences between these values and the theoretical ones. It is also observed in Figure $1 \mathrm{~b}$ that there is an increase in the $\mathrm{C} 1 \mathrm{~s}$ concentration (58.6 atomic \%) when compared to the S1 reference coating in Figure 1a, but this is associated with a substantial change in the $\mathrm{C} 1 \mathrm{~s}$ peak shape. In Figure $1 \mathrm{~b}$ the $\mathrm{C} 1 \mathrm{~s}$ peak now possesses a more intense lower binding energy component peak than in Figure 1a, indicating an increase in the hydrocarbon component of the S2 coating. This increase in the intensity of the hydrocarbon component peak is accompanied by a reduction in the intensity of the component peak at higher binding energy $\left(\mathrm{CF}_{2}\right)$ in the $\mathrm{C} 1 \mathrm{~s}$ peak. Although the component peak at higher binding energy in the $\mathrm{C} 1 \mathrm{~s}$ peak is still clearly observed in Figure $1 \mathrm{~b}$, it is not as distinct as in Figure 1a.

The decrease in F1s concentration, reduction in intensity of the $\mathrm{CF}_{2}$ component of the $\mathrm{C} 1 \mathrm{~s}$ peak, the increase in $\mathrm{O} 1 \mathrm{~s}$ concentration over and above the theoretical value and the greater intensity of the hydrocarbon component of the $\mathrm{C} 1 \mathrm{~s}$ peak in Figure $1 \mathrm{~b}$ 
are all attributed to the surface segregation of the acrylic flow agent included in the S2 coating formulation. See Table 3 for the theoretical values for the flow agent. It is well documented that the addition of a flow agent to a coating formulation alters the surface composition and chemistry of a coating [24], due to the formation of a flow agent segregation layer. Such flow agent segregation layers are typically $\sim 1 \mathrm{~nm}$ thick [25]. This XPS data suggests that the F1s signal is being attenuated by the formation of a flow agent segregation layer at the coating surface. That is, for the S2 coating in Figure $1 \mathrm{~b}$, the addition of the acrylic flow agent not only results in changes in the chemical and material composition of the surface but also leads to an attenuation of the XPS signal associated with the fluorine in the PVdF, indicating that the PVdF component of the coating is buried below a flow agent segregation layer in the S2 coating.

In Figure 1c C1s, O1s and F1s XPS peaks are again observed, this time for the S3 coating formulation (S1 reference formulation plus acrylic copolymers). In Figure 1c a substantial decrease in the intensity of the F1s peak (from 45.9 to 15.4 atomic \%) is now observed, when compared to the $\mathrm{S} 1$ coating. This is accompanied by a large increase in the intensity of the O1s peak (from 2.6 to 16.4 atomic \%). There is also an increase in the intensity of the $\mathrm{C} 1 \mathrm{~s}$ peak (from 51.5 to 68.3 atomic \%) when compared to the S1 coating in Figures 1a (see Table 2). Perhaps more noticeable in Figure 1c is the change in the C1s peak shape. Strong attenuation of the F1s signal in the $\mathrm{S} 3$ coating results in a loss of the distinct $\mathrm{CF}_{2}$ component peak at higher binding energy (as observed in Figures 1a and $1 \mathrm{~b}$ for the S1 and S2 coatings) in the C1s XPS 
peak. The $\mathrm{CF}_{2}$ component peak is now only observed as part of the asymmetry to the higher binding energy side of the carbon peak.

When compared to the theoretical values the carbon concentration is $40 \%$ higher (68.3 vs. 48.9$)$ where as that of oxygen is only $30 \%$ higher (16.4 vs. 12.1). This could be explained by a higher PMMA concentration at the surface caused by some surface segregation. The excess carbon could be due to adsorption of adventitious carbon containing contaminants. Thus like the flow agent added to the S2 coating formulation, the acrylic copolymers (or a substantial fraction of them) segregate to the $\mathrm{S} 3$ coatings air/coating surface. This segregation of the acrylic copolymers both dramatically changes the material and chemical composition of the S3 coatings air/coating surface and results in the strong attenuation of the F1s signal. The attenuation of the F1s XPS signal is due to the formation of an acrylic copolymer segregation layer at the S3 coatings air/coating surface. Given that the decrease in F1s intensity observed for the S3 coating in Figure 1c is greater than that observed for the $\mathrm{S} 2$ coating in Figure $1 \mathrm{~b}$ it is reasonable to assume that the acrylic copolymer segregation layer formed for the S3 coating is thicker than the segregation layer formed by the acrylic flow agent in the S2 coating.

For the S4 coating the flow agent used to produce the S2 coating and the acrylic copolymers included in the S3 coating were added to the S1 reference coating formulation. The 250-700 eV region of an XPS survey spectrum of the S4 coating is presented in Figure 1d. The F1s peak (0.1 atomic \%) is now so heavily attenuated 
when compared to the F1s peaks in Figures $1 \mathrm{a}, 1 \mathrm{~b}$ and 1c that the F1s XPS peak is now barely observed in the S4 coating survey spectrum in Figure 1d. The intensity of the O1s peak (18.8 atomic \%) in Figure 1d is greater than that observed for the S3 coating in Figure 1c. There is a further substantial increase in the $\mathrm{C} 1 \mathrm{~s}$ concentration (81.1 atomic \%) and hence peak intensity in the S4 coating in Figure 1d. The C1s peak shape now shows no discernible contribution from the $\mathrm{CF}_{2}$ component peak associated with the PVdF component of the formulation and the peak shape in Figure $1 \mathrm{~d}$ is now that of a dominant hydrocarbon peak with some minor asymmetry to the higher binding energy side of the peak. The lack of an F1s peak in Figure 1d indicates that the PVdF component of the S4 coating is now buried beneath a significant segregation layer. The concentration ratios are now very similar to those that would be observed from the pure acrylic flow aid, indicating that the surface consists largely of a fairly thick layer of the flow aid. The analysis depth of XPS is generally taken to approximate to $3 \lambda$ (where $\lambda$ is the electron attenuation length of the material being investigated), although one must remember that the electron intensity decays exponentially within this sampling volume, in accord with the well known BeerLambert formalism. Frey et al have demonstrated that $\lambda$ for a fluorinated material is 3-4 nm [26]. The total thickness of this segregation layer (flow aid plus acrylic copolymer) in the $\mathrm{S} 4$ coating is great enough $(<<10 \mathrm{~nm})$ to almost completely mask the PVdF component of the coating. This suggests the segregation layer must be at least $\geq 8 \mathrm{~nm}$ thick i.e. occupying most if not all of the XPS analysis depth. The very small amount of $\mathrm{F}$ emanates not from the underlying PVdF coating but from a very small amount of fluorinated material that is incorporated into the segregated layer. 
The XPS spectra in Figures 1a-d suggest that both the acrylic flow agent included in the $\mathrm{S} 2$ coating and the acrylic copolymers included in the S3 coating have a tendency to segregate towards the coating's air/coating surface. However, the flow agent is designed to segregate to the coating surface, by virtue of its incompatibility, and thus aids the coating aesthetics by equilibrating the surface free energy areas that vary in this quantity. It has previously been demonstrated that the air/coating surface of a fully formulated PVdF coating is dominated by the acrylic flow agent even when acrylic copolymers are included in the formulation [27]. It is therefore reasonable to propose that the $\mathrm{S} 4$ coating surface is multilayer. That is, the air/coating surface of the S4 coating is composed of a flow agent segregation layer, beneath this is a subsurface layer either formed by, or rich in, the segregating acrylic copolymers. Finally, there is the coating bulk, which presumably possesses a homogeneous PVdF/acrylic copolymer composition. However, this hypothesis of a multilayer coating surface cannot be easily validated using the XPS results. To validate this hypothesis depth profiling of the PVdF coating is desired.

In Figure 2 negative ion elemental depth profiles from the outermost nanometres of the S4 coating's air/coating surface are presented. Variations in the intensity (and thus concentration) of $\mathrm{O}$ and $\mathrm{F}$ have been followed with respect to etch time and thus depth into the $\mathrm{S} 4$ coating. The $\mathrm{O}$ and $\mathrm{F}$ depth profiles in Figure 2 were obtained using $\mathrm{Bi}_{3}{ }^{+}$cluster ions from a Bi liquid metal ion source as the analysis source and $\mathrm{C}_{60}{ }^{+}$ions from a Buckminsterfullerene $\left(\mathrm{C}_{60}\right)$ source as the etch source. $\mathrm{A}_{60}{ }^{+}$etch interval of 
$10 \mathrm{~ms}$ was employed. Szakal et al have previously demonstrated that employing a $\mathrm{C}_{60}$ source for etching enables chemical and molecular specificity to be maintained when etching through polymeric materials such as PMMA [22].

An apparent disparity exists between the XPS results and the ToF-SIMS depth profile results which is immediately obvious to any one but the casual observer! That is the XPS results predict zero or almost zero fluorine in the top few nanometers of the surface of S4 and only once the flow aid layer/acrylic copolymer layer has been eroded by the $\mathrm{C} 60$ source should the PVdF be revealed. Thus a zero level plateau should be observed in Figure 2a for the first 0.5 to 1 s of erosion time (assuming 4-8 $\mathrm{nm}$ of erosion per second). However, given the differences in the elemental cross sections (the detection limit for fluorine in ToF-SIMS is far superior to that in XPS) and the analysis depths for XPS and SIMS ( $10 \mathrm{~nm}$ and $1 \mathrm{~nm}$ respectively) it is possible that a F concentration of 0.1 atomic $\%$ as measured by XPS at the S4 coating surface is equal to an F intensity of $\sim 7 \times 10^{-2}$ counts/s as observed in SIMS. Additionally, it is likely that the $\mathrm{Bi}_{3}{ }^{+}$ions used for the initial analysis removes a substantial portion of the loosely bound low molecular weight flow aid, which is sitting on the surface, resulting in the phenomena observed.

The concentration of $\mathrm{F}$ is seen to increases steadily with depth through the subsurface region of the coating ( $0-1 \mathrm{~s}$ etch) in Figure $2 \mathrm{a}$. The concentration of $\mathrm{F}$ in the depth profile in Figure 2a then continues to increase but at a slower rate (between 1 and $2.5 \mathrm{~s}$ etch) before reaching a stable concentration (after $2.5 \mathrm{~s}$ etch) in the $\mathrm{S} 4$ 
coating bulk. That the intensity of the F depth profile is at a minimum at the air/coating surface of the S4 coating is consistent with the interpretation of the XPS data in Figures 1-4 which indicated that the S4 coating's air/coating surface is composed of the acrylic flow agent. That the F intensity increases in the sub-surface region of the S4 coating (0.1-2 s etch) is also consistent with the XPS data in Figures 1a-d in that the sub-surface region is rich in the acrylic copolymers included in the formulation but this acrylic rich layer has a finite thickness.

The shape of the $\mathrm{O}$ depth profile in Figure $2 \mathrm{~b}$ is substantially different to that of the $\mathrm{F}$ depth profile in Figure 2a. It is observed in Figure $2 b$ that the $O$ depth profile exhibits an intensity maximum close to the coating surface (within $0.25-0.4 \mathrm{~s}$ etch). The $\mathrm{O}$ intensity then gradually decreases with etch time and thus depth until the $\mathrm{O}$ intensity stabilises in the bulk of the $\mathrm{S} 4$ coating (after $2.5 \mathrm{~s}$ etch). The shape of the O depth profile in Figure $2 b$ suggests that the surface and sub-surface regions of the S4 coating are rich in O containing materials, when compared to the bulk of the S4 coating (the region beyond $2.5 \mathrm{~s}$ etch in Figure $2 \mathrm{~b}$ ). Given that the acrylic copolymers have a greater oxygen content than the flow aid (see Table 3), the O profile is in keeping with the interpretation of the XPS results, which suggest the formation of a flow agent surface layer over an acrylic copolymers rich subsurface segregation layer in the S4 coating. Such segregation layers would be expected to be rich in $\mathrm{O}$ when compared to the S4 coating bulk, as is observed in Figure $2 \mathrm{~b}$. 
In the sub-surface region of the $\mathrm{S} 4$ coating the $\mathrm{F}$ concentration in the $\mathrm{F}$ depth profile in Figure 2a is increasing with depth while that in the $\mathrm{O}$ depth profile in Figure $2 b$ reaches a concentration maximum in the sub-surface region of the $\mathrm{S} 4$ coating. The stability of the intensity of the $\mathrm{O}$ and $\mathrm{F}$ depth profiles after $2.5 \mathrm{~s}$ etch in Figure 2 suggests that the S4 coating bulk possesses a homogeneous material composition. This stability of the material composition of the S4 coatings bulk contrasts sharply with the material composition of the sub-surface region of the S4 coating, which exhibits considerable variation. This variation in material composition in the subsurface is due to materials included in the coating formulation segregating towards the S4 coating's air/coating surface. This tendency of components included in the coating formulation to segregate towards the S4 coating's air/coating surface is driven by the differences in the compatibility of the different components that make up the formulation.

To further investigate the possible formation of an acrylic co-polymers rich subsurface layer in the S4 coating, positive ion molecular depth profiles were obtained. The molecular depth profiles in Figures 3 and 4 were obtained in the manner previously described. In Figure 3 positive ion depth profiles of the molecular fragments $\mathrm{C}_{3} \mathrm{HF}_{4}{ }^{+}, \mathrm{C}_{5} \mathrm{H}_{2} \mathrm{~F}_{5}{ }^{+}$, and $\mathrm{CF}_{3}{ }^{+}$, diagnostic of the $\mathrm{PVdF}$ component of the $\mathrm{S} 4$ coating [28,29], are presented. It is observed in Figure 3 that the depth profiles for the $\mathrm{C}_{3} \mathrm{HF}_{4}{ }^{+}, \mathrm{C}_{5} \mathrm{H}_{2} \mathrm{~F}_{5}{ }^{+}$, and $\mathrm{CF}_{3}{ }^{+}$molecular ions originating from the $\mathrm{PVdF}$ are identical in shape to the F depth profile in Figure 2a (although of varying intensity). All of the depth profiles presented in Figure 3 exhibit an increasing F intensity with depth in the 
surface and sub-surface regions of the $\mathrm{S} 4$ coating. This is followed by a region of stable F intensity in the S4 coating bulk.

In Figure 4 positive ion molecular depth profiles of the fragments, $\mathrm{C}_{4} \mathrm{H}_{5} \mathrm{O}^{+}, \mathrm{C}_{3} \mathrm{H}_{5} \mathrm{O}^{+}$, and $\mathrm{C}_{8} \mathrm{H}_{11} \mathrm{O}_{2}{ }^{+}$, diagnostic of the acrylic copolymers included in the $\mathrm{S} 4$ coating formulation are presented [30]. It is noted that the shapes of the depth profiles in Figure 4 are similar to those observed for the $\mathrm{O}$ depth profile in Figure $2 \mathrm{~b}$. All of the depth profiles in Figure 4 exhibit an intensity maximum in the sub-surface region of the $S 4$ coating $(0.35-0.45 \mathrm{~s}$ etch). The intensity of the depth profiles in Figure 4 then falls gradually with etch time and thus depth into the coating (0.5-1.0 s etch). The depth profiles in Figure 4 then assume more stable intensity values in the bulk of the S4 coating (beyond $1.0 \mathrm{~s}$ etch). The shape of the acrylic copolymer derived depth profiles in Figure 4 reveals that the $\mathrm{O}$ rich sub-surface layer suggested by the XPS data and the elemental depth profiles does indeed result from the segregation of the acrylic copolymers towards the S4 coating's air/coating surface.

This new information concerning the surface and subsurface morphologies will have an impact on the interpretation of durability as measured by gloss retention and certainly opens the way for investigations into how the surface changes after weathering in specific environments and locations. Furthermore the thick layer of flow agent and acrylic co-polymer may explain why the well documented resistance of PVdF coatings to dirt pick up is not as good as might be anticipated from a clean PVdF surface. Precise quantification of the layer thickness may help in determining 
how an inter-phase could be set up if it is desired to overcoat the PVdF. That is fluorine polymers are notoriously difficult to wet out, hence the chances of a good inter-phase of over 50nm being set up are small resulting in poor inter-coat adhesion. Knowledge of how to alter the composition of the near surface region could help in the design of a third-coat.

The elemental and molecular ion depth profiles in Figures 2, 3 and 4 provide confirmatory evidence for the formation of an acrylic copolymer rich layer within the sub-surface region of the S4 coating, resulting from segregation of the acrylic copolymers towards the S4 coating's air/coating surface. The XPS analyses of the S1S4 coating formulations and the elemental and molecular ion SIMS depth profiles of the S4 coating all indicate that the S4 coating's outermost nanometres are composed of three distinct layers. The S4 coating's air/coating surface is composed of the acrylic flow agent included in the S2 and S4 coating formulations. It has previously been demonstrated by Perruchot et al that flow agent layers are typically $0.5-1.0 \mathrm{~nm}$ thick [25], depending on the nature and concentration of the flow agent employed. Below this flow agent layer is a sub-surface layer rich in the acrylic co-polymer included in the S3 and S4 coating formulations. The acrylic copolymers rich subsurface layer is the result of the segregation of the acrylic co-polymers towards the S4 coating's air/coating surface. Although it might be viewed that the flow agent and acrylic copolymers are both segregating to the S4 coating's air/coating surface, this is a competitive process. The levelling capability of the flow agent is better than that of the acrylic copolymers and so it is the flow agent that reaches the air/coating surface, 
probably due to its molecular weight differences causing incompatibility. Some of the more compatible acrylic co-polymers may segregate towards the coating's air/coating surface alongside the flow agent, leading to the proposed structure of the near-surface region, which is somewhat different to that suggested by Gu et al who apparently observed a fluorine rich surface [31]. The segregating acrylic copolymers are therefore unable to reach the air/coating surface and thus form an acrylic co-polymers rich sub-surface layer within the S4 coating. The final layer in the coating is the S4 coating bulk, which all analyses suggest possesses a homogeneous material composition.

\section{Conclusions:}

The XPS and TOF-SIMS depth profiling characterisation of the outermost nanometres of a PVdF/acrylic copolymers coating based on a 'real world' commercial formulation has been demonstrated. The XPS analyses reveal that both the flow agent and acrylic copolymers included in the formulation segregate towards the coating's air/coating surface. Addition of flow agent or acrylic copolymers to the coating formulation leads to attenuation of the F1s XPS signal. When both the flow agent and acrylic co-polymers are added to the same coating formulation the segregation layer may be sufficiently thick to mask the F1s signal from the underlying PVdF component of the formulation. The XPS results suggest the more fully formulated coating (S4) possesses an acrylic rich layer at the surface and in the sub-surface region of the coating. 
We have demonstrated that a $\mathrm{Bi}$ analysis source and a $\mathrm{C}_{60}$ etch source can be used in combination to obtain elemental and molecular SIMS depth profiles from complex $\mathrm{PVdF}$ based coating formulations. The $\mathrm{O}$ depth profile revealed the existence of an oxygen rich layer in the sub-surface region of the S4 coating. The F depth profile showed that the surface and sub-surface regions of the coating were depleted in $\mathrm{F}$ when compared to the bulk of the coating. SIMS positive ion molecular depth profiles revealed that the sub-surface region of the $\mathrm{S} 4$ coating is rich in the acrylic copolymers included in the coating formulation. These results confirm the segregation of the acrylic co-polymers towards the air/coating surface of the $\mathrm{S} 4$ coating. Overall the XPS and SIMS depth profiling results suggest the outermost nanometres of the S4 coating is composed of three distinct layers; a thin flow agent layer at the air/coating surface, an acrylic co-polymer rich sub-surface layer and the coating bulk which possesses a homogeneous material composition. 


\section{References:}

1) J. Scheirs, S. Burks, A. Locaspi, Trends Polym. Sci. 3 (1995) 74.

2) H.R. Thomas, J.J. OMalley, Macromolecules 12 (1979) 323.

3) W. Lee, W.Cho, C. Ha, A. Takahara, T. Kajiyama, Polymer 36 (1995) 1229.

4) L. Ying, E.T. Kang, K.G. Neoh, K.Kato, H. Iwata, J. Membrane Sci. 243 (2004) 253.

5) M. Momtaz, J-L. Dewez, J. Marchand-Brynaert, J. Membrane Sci. 250 (2005) 29.

6) J. Mueller, R.H. Davis, J. Membrane Sci. 116 (1996) 47.

7) D. Klee, Z. Ademovic, A. Bosserhoff, H. Hoecker, G. Maziolis, H-J Erli, Biomaterials 24 (2003) 3663.

8) Z. Ademovic, A. Gonera, P. Mischnick D. Klee, Biomacromolecules 7 (2006) 1429.

9) Z. Ademovic, D. Klee, P. Kingshott, R. Kaufmann, H. Höcker, Bimolecular Eng. 19 (2002) 177.

10) S.J. Hinder, C. Lowe, J.T. Maxted, J.F. Watts, Prog. Org. Coat. 54 (2005) 20.

11) S.J. Hinder, C. Lowe, J.T. Maxted, J.F. Watts, Prog. Org. Coat. 54 (2005) 104.

12) S.J. Hinder, C. Lowe, J.T. Maxted, J.F. Watts, Surf. Interface Anal. 36 (2004) 1575 .

13) E.R. Fuoco, G. Gillen, M.B.J. Wijesundara, W.E. Wallace, L. Hanley. J. Phys. Chem. B 105 (2001) 3950.

14) K. Norrman, K.B. Haugshøj, N.B. Larsen. J. Phys. Chem. B 106 (2002) 13114.

15) C.M. Mahoney, S.V. Robertson, G. Gillen. Anal. Chem. 76 (2004) 3199.

16) C.M. Mahoney, J. Yu, J.A. Gardella Jr. Anal. Chem. 77 (2005) 3570.

17) J. Cheng, N. Winograd. Anal. Chem. 77 (2005) 3651

18) A.G. Sostarecz, S. Sun, C. Szakal, A. Wucher, N. Winograd. Appl. Surf. Sci. 231232 (2004) 179. 
19) A.G. Sostarecz, C.M. McQuaw, A. Wucher, N. Winograd. Anal. Chem. 76 (2004) 6651.

20) A. Wucher, S. Sun, C. Szakal, N. Winograd. Appl. Surf. Sci. 231-232 (2004) 68.

21) A. Wucher, S. Sun, C. Szakal, N. Winograd. Anal. Chem. 76 (2004) 7234.

22) C. Szakal, S. Sun, A.Wucher, N. Winograd. Appl. Surf. Sci. 231-232 (2004) 183.

23) G. Beamson, D. Briggs, High Resolution XPS of Organic Polymers; The Scienta ESCA300 Database, John Wiley \& Sons Ltd: Chichester, (1992) 228.

24) S.R. Leadley, J.F. Watts, C.J. Blomfield, C. Lowe, Surf. Interface Anal. 26 (1998) 444.

25) C. Perruchot, J.F. Watts, C. Lowe, R.G. White, P.J. Cumpson, Surf. Interface Anal. 34 (2002) 570.

26) S. Frey, K. Heister, M. Zharnikov, M. Grunze, K. Tamada, R. Colarado Jr. Isr. J. Chem. 81-97 (2000) 40.

27) S.J. Hinder, C. Lowe, J.F. Watts, Accepted for publication in Surf. Interface Anal. 2006.

28) J. Feng, C-M. Chan, L-T. Weng, Polymer 41 (2000) 2695.

29) P. Spevack, Y. Deslandes, Appl. Surf. Sci. 99 (1996) 41.

30) A.M. Leeson, M.R.Alexander, R.D. Short, D. Briggs, M.J. Hearn, Surf. Interface Anal. 25 (1997) 261.

31) X. Gu, C.A. Michaels, D. Nguyen, Y.C. Jean, J.W. Martin, T. Nguyen, App. Surf. Sci. 252 (2006) 5168. 
a)

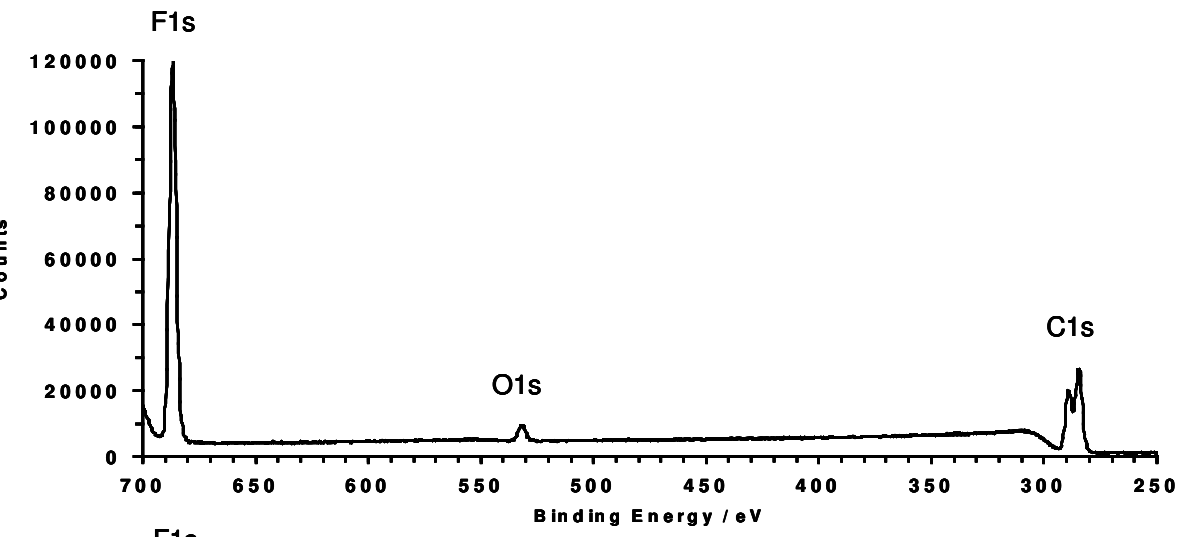

b)

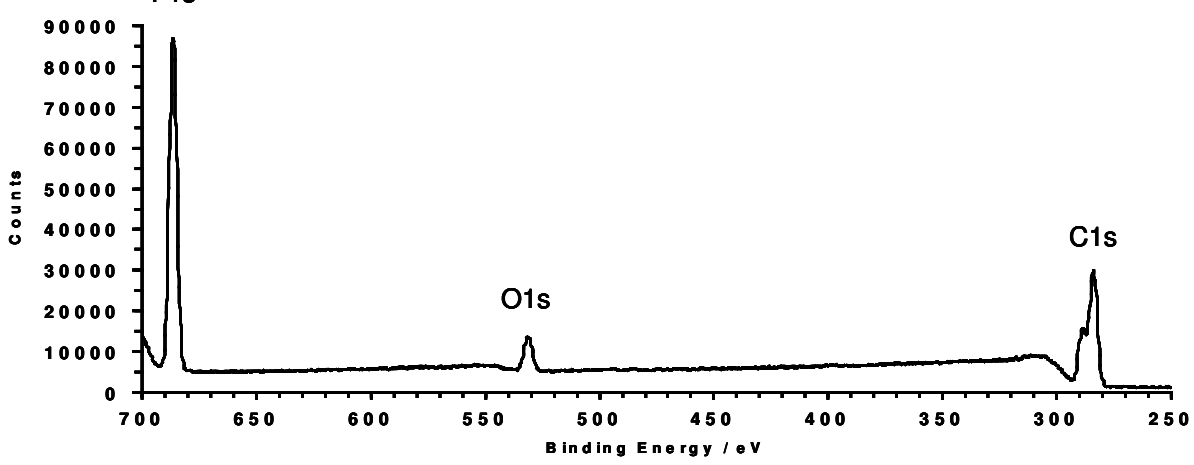

c)
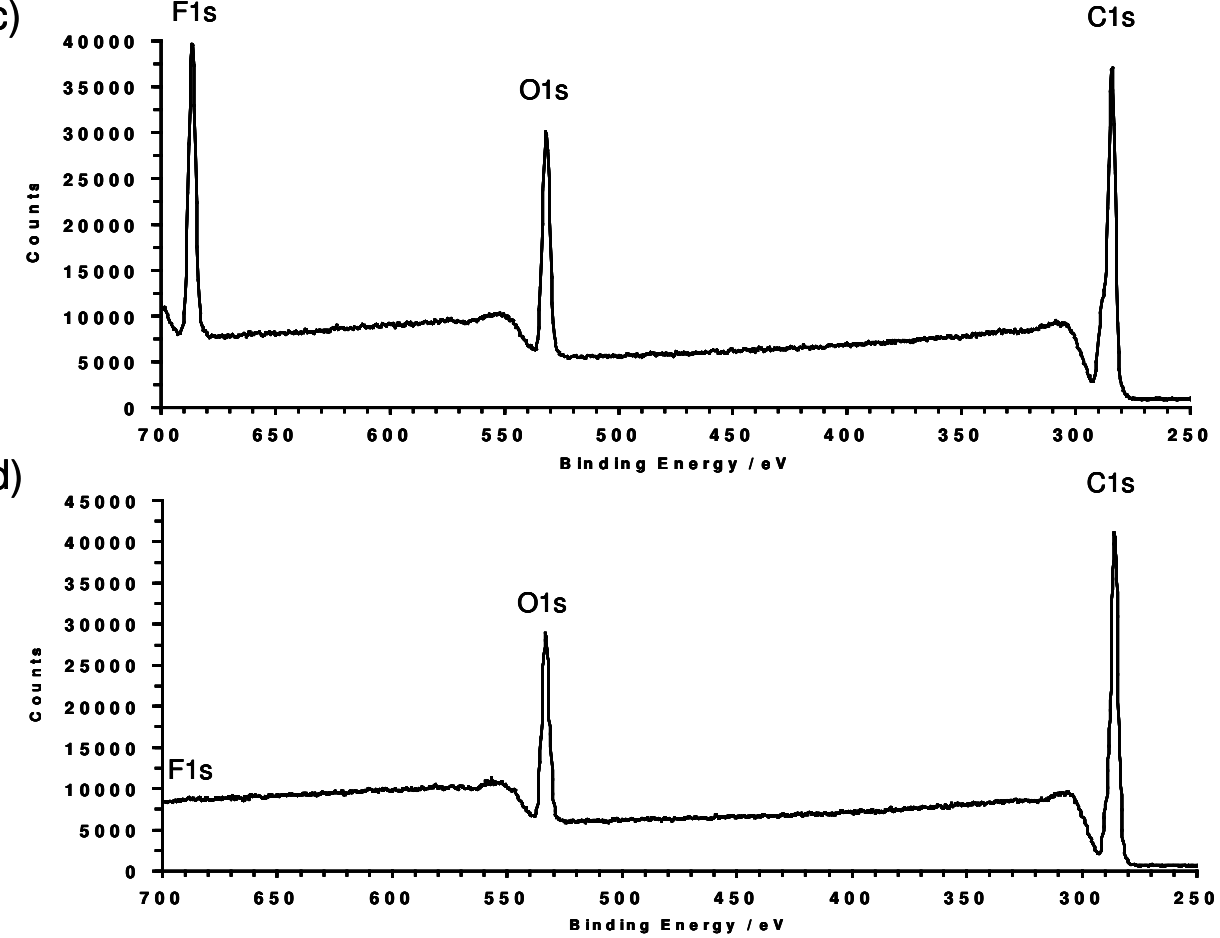

Figure 1. The $250-700 \mathrm{eV}$ region of a series of XPS survey spectra of a) S1 coating (reference formulation), b) S2 coating (S1 reference formulation plus flow agent),

c) S3 coating ( $\mathrm{S} 1$ reference formulation plus acrylic co-polymers) and d) S4 coating (S1 Reference formulation plus flow agent and acrylic co-polymers). 
a)

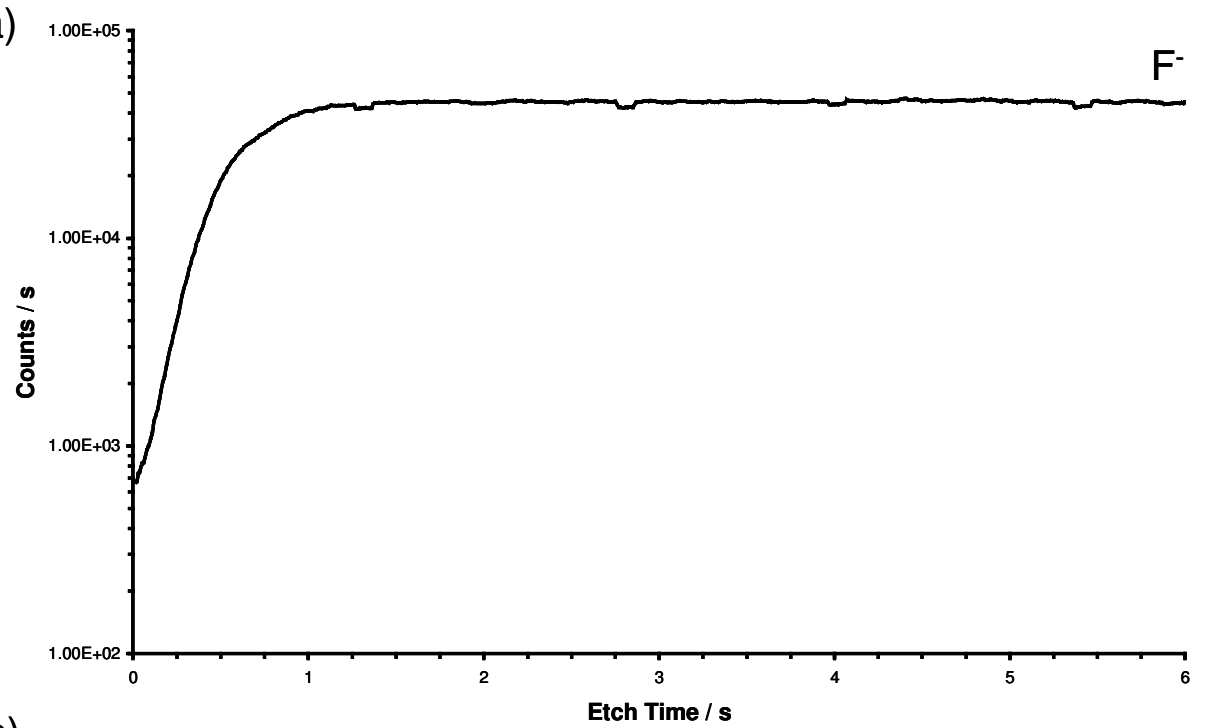

b)

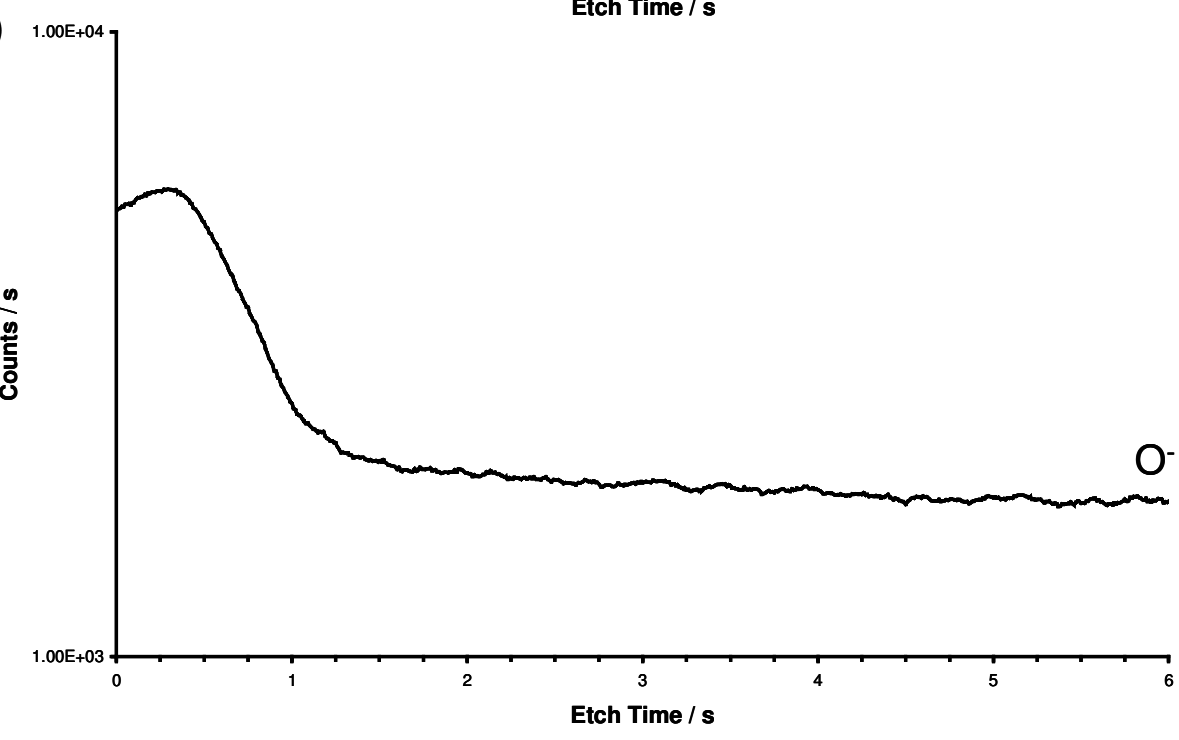

Figure 2. ToF-SIMS negative ion mode, elemental depth profiles for a) fluorine and b) oxygen. The depth profiles were obtained from the air/coating surface of the S4 coating sample. 


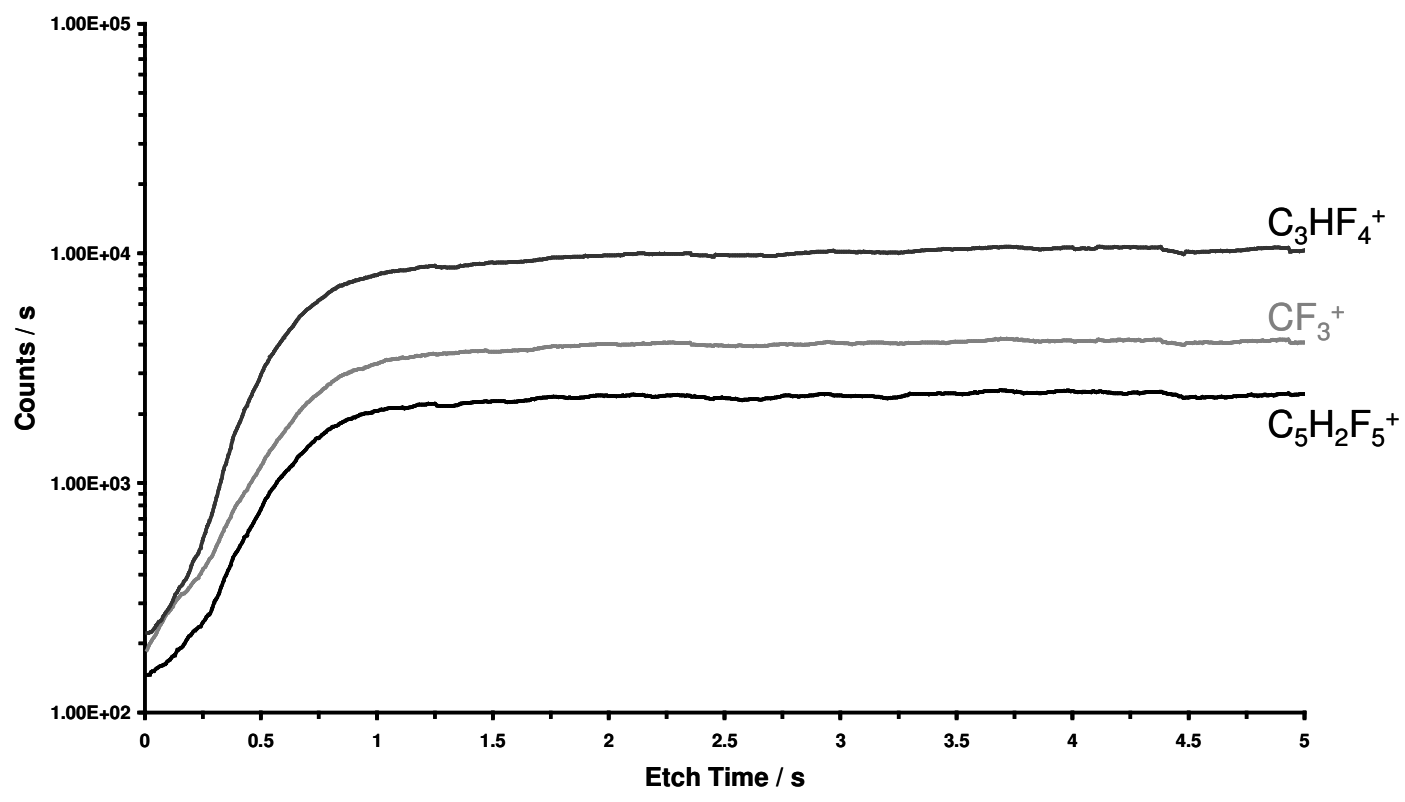

Figure 3. ToF-SIMS positive ion mode, molecular depth profiles for the $\mathrm{C}_{3} \mathrm{HF}_{4}{ }^{+}, \mathrm{CF}_{3}{ }^{+}$ and $\mathrm{C}_{5} \mathrm{H}_{2} \mathrm{~F}_{5}{ }^{+}$fragments characteristic of $\mathrm{PVdF}$. The depth profiles were obtained from the air/coating surface of the $\mathrm{S} 4$ coating sample. 


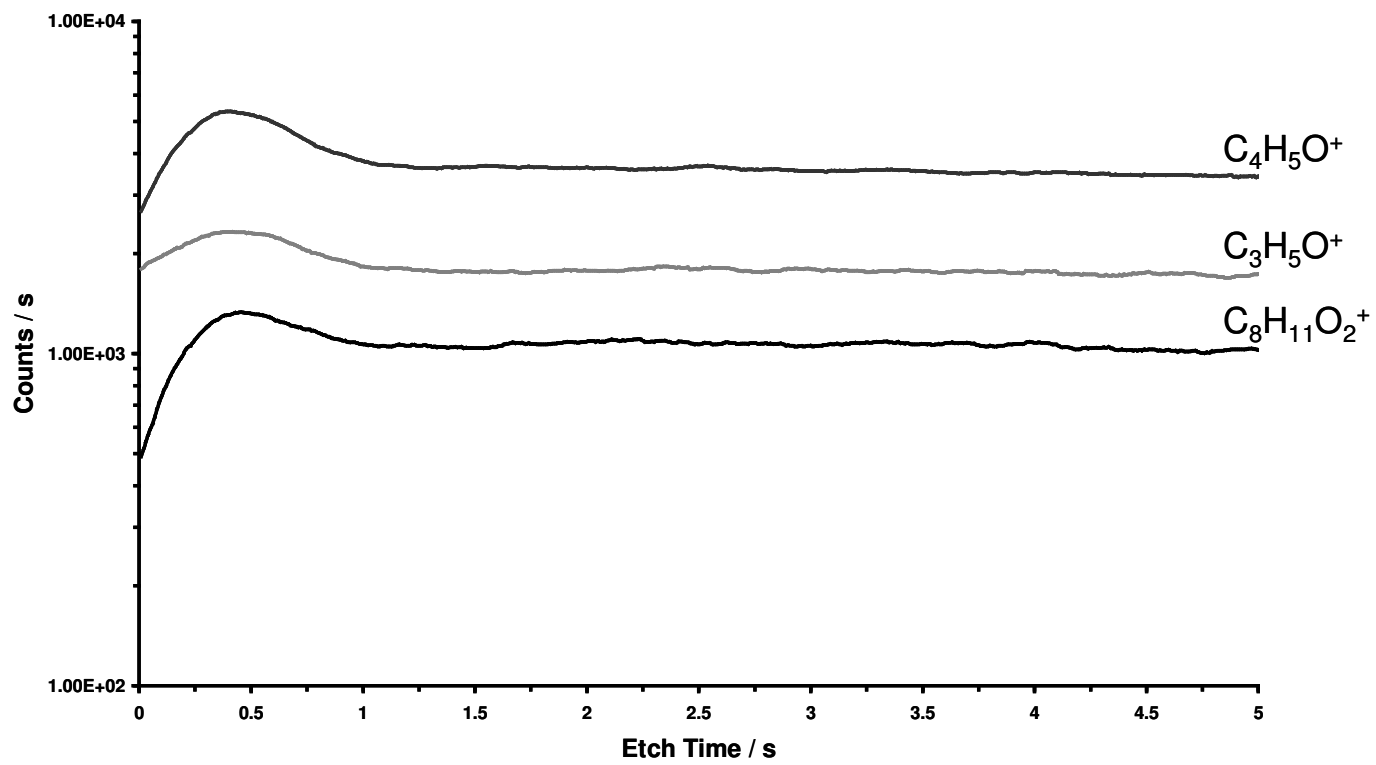

Figure 4. ToF-SIMS positive ion mode, molecular depth profiles for the $\mathrm{C}_{4} \mathrm{H}_{5} \mathrm{O}^{+}$, $\mathrm{C}_{5} \mathrm{H}_{9} \mathrm{O}^{+}$and $\mathrm{C}_{8} \mathrm{H}_{11} \mathrm{O}_{2}^{+}$fragments characteristic of the acrylic co-polymers included in the $\mathrm{S} 4$ coating formulation. The depth profiles were obtained from the air/coating surface of the S4 coating sample. 


\begin{tabular}{|l|l|}
\hline Coating & \multicolumn{1}{l|}{ Formulation } \\
\hline S1 & PVdF Topcoat Reference Formulation \\
\hline S2 & As in S1 + flow agent. \\
\hline S3 & As in S1 + acrylic co-polymers \\
\hline S4 & As in S1 + flow agent and acrylic copolymers. \\
\hline
\end{tabular}

Table 1. PVdF coating sample formulations. 


\begin{tabular}{|l|c|c|c|c|c|c|}
\hline \multirow{2}{*}{ Coating } & \multicolumn{6}{|c|}{ Surface Composition/Atomic \% } \\
& C1s & F1s & \multicolumn{2}{c|}{ O1s } \\
\hline & Actual & Theory & Actual & Theory & Actual & Theory \\
\hline S1 & 51.5 & 42.0 & 45.9 & 55.5 & 2.6 & 2.5 \\
\hline S2 & 58.6 & 43.4 & 36.3 & 53.4 & 5.1 & 3.2 \\
\hline S3 & 68.3 & 48.9 & 15.4 & 39.0 & 16.4 & 12.1 \\
\hline S4 & 81.1 & 49.6. & 0.1 & 37.0 & 18.8 & 12.2 \\
\hline
\end{tabular}

Table 2. XPS and theoretical (from formulation) quantification data of the PVdF coating samples analysed. 


\begin{tabular}{|l|c|c|c|}
\hline Material & C1s & F1s & O1s \\
\hline PVdF & 39.2 & 61 & 0 \\
\hline Plasticiser & 71.3 & 0 & 28.7 \\
\hline Flow Agent & 80.9 & 0 & 19.1 \\
\hline Acrylic Co-polymer & 66.3 & 0 & 33.3 \\
\hline
\end{tabular}

Table 3. Theoretical values for compositions of components used in coatings formulations. 